Engineering and Computational Mechanics Volume 169 Issue EM2

Perforated tunnel exit regions and micro-pressure waves: geometrical influence

Wang, Vardy and Pokrajac
Proceedings of the Institution of Civil Engineers

Engineering and Computational Mechanics 169 June 2016 Issue EM2 Pages 70-85 http://dx.doi.org/10.1680/jencm.15.00026 Paper 1500026

Received 10/11/2015 Accepted 03/02/2016

Published online 02/03/2016

Keywords: computational mechanics/fluid mechanics/noise ice

$\overline{\text { Institution of Civil Engineers }}$

\title{
Perforated tunnel exit regions and micro-pressure waves: geometrical influence
}

1 Honglin Wang MSC, PhD

School of Mechanical Engineering, Southwest Jiaotong University, Chengdu, China

2 Alan E. Vardy DEng, DSC, FREng, FRSE

Research Professor, School of Science and Engineering, University of

Dundee, Dundee, UK (corresponding author: a.e.vardy@dundee.ac.uk)
3 Dubravka Pokrajac MEng, MSc, PhD

Professor, School of Engineering, University of Aberdeen, Aberdeen, UK
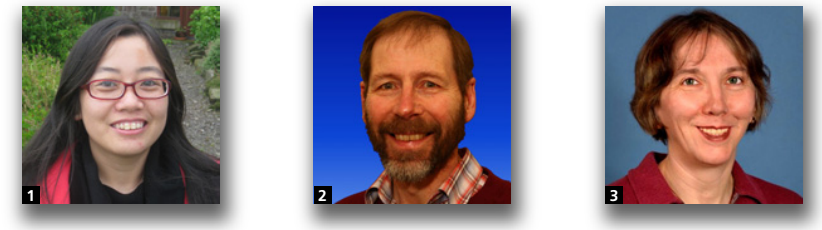

The effectiveness of long, perforated exit regions in reducing the radiation of micro-pressure waves (MPWs) from railway tunnels is assessed. Such disturbances always occur, but their amplitudes are usually small. For the particular case of high-speed trains, they can reach levels that would cause annoyance in the absence of suitable countermeasures. This risk is especially large in the case of long tunnels. The general behaviour of wave reflection/ transmission/radiation at a perforated exit region has been explored in previous papers that have (a) quantified the dependence on the characteristics of the incident wavefront reaching the exit region from further upstream in the tunnel and $(b)$ validated the numerical methodology in a searching manner. Some notable differences have been found in comparison with the criteria that have long been known for unperforated exit regions. In particular, the resulting MPW amplitudes depend on the amplitudes of incident wavefronts as well as on their steepnesses. This paper summarises these outcomes and uses the methodology to explore important practical design issues, namely the dependence of the effectiveness of perforated exit regions on their length and cross-sectional area. Once again, differences are found from the behaviour of unperforated regions.

\section{Introduction}

Many papers have discussed the mitigation of micro-pressure waves (MPWs) emitted from railway tunnel portals (e.g. Ozawa and Maeda, 1988; Raghunathan et al., 2002). A wide range of possible countermeasures exist, but by far the most commonly implemented method is the provision of an extended entrance region to reduce the steepness of pressure wavefronts generated during train entry (e.g. Howe et al., 2003; Ozawa et al., 1991). Such entrances have little influence on the maximum amplitudes of train-entry wavefronts, but they can greatly extend the time taken to reach the maxima. This is important because the rates of change of pressure associated with wavefronts inside tunnels have a crucial influence on the amplitudes of emitted MPWs (e.g. Ozawa and Maeda, 1988).
Notwithstanding the widespread success of extended entrance regions, they are not always the most suitable countermeasure. In slab-track tunnels, for instance, the effectiveness of entrance regions decreases with increasing tunnel length because wavefronts steepen as they propagate along such tunnels (e.g. Fukuda et al., 2006). It is even possible for entrance regions to have almost no benefit at all. An obvious example is when the wavefront causing the greatest MPW is generated when a train is already well inside a tunnel - at a large shaft, perhaps. When the suitability of entrance regions is not assured, attention needs to be turned to other countermeasures. In general, these can include attacking the wave as it propagates along the tunnel itself (e.g. Fukuda et al., 2001; Sockel and Pesave, 2006; Sugimoto, 1993) or influencing the way in which it behaves at the tunnel exit (e.g. Aoki et al., 1999; Kim et al., 2004; 
Matsubayashi et al., 2004). This paper explores some characteristics of one particular method, namely extended exit regions that, at first sight, are closely similar to conventional entrance regions.

Before focusing exclusively on exit regions, it is useful to draw attention to reasons why they have hitherto been considered to be less useful than entrance regions of equal length. In a nutshell, this is because the fundamental time scale for an entrance region is the difference between the times needed for (a) a train nose and $(b)$ a sound wave to travel along the region. For train speeds below a Mach number of $0 \cdot 5$, the leading edge of the nose-entry wavefront propagates at more than twice the speed of the train and so the overall length of the eventual nose-entry wavefront exceeds the length of the region. In contrast, an exit region must serve its purpose long before the train arrives so its fundamental time scale is the (usually smaller) time needed for a wave to travel along it. This inherent advantage of entrance regions will inevitably reduce if train speeds continue to increase as they have done in recent decades. Even at present-day speeds, the advantages can vanish in long slab track tunnels due to the inertial steepening between the entrance and the exit. Accordingly, it is appropriate to explore alternative methods of countering MPWs and perforated exit regions are obvious candidates for consideration.

In previous papers, the authors have assessed simple exit regions with uniformly distributed openings to atmosphere along their lengths. First, in Wang et al. (2015a), a rigorous numerical methodology was developed and was used to deduce the characteristic response of a perforated exit region to wavefronts arriving along the tunnel. The principal purpose of the paper was to establish a reliable numerical method. Only one exit region was considered and this was a simple, but indicative configuration with uniformly distributed openings along an exit region with the same cross-section as the tunnel. Furthermore, a planar two-dimensional (2D) geometry was chosen, thereby limiting the direct applicability of the predictions to deep, cut-and-cover tunnels. Notwithstanding these simplifications, the paper identified important characteristics of wavefronts propagating along perforated exit regions and their consequences for MPW generation. In particular, it showed that the emitted MPWs are influenced by the amplitudes of internal wavefronts as well as by their steepnesses. This seemingly obvious result is actually in stark contrast with the corresponding behaviour of unperforated regions (i.e. conventional tunnel exits). In such cases, for wavefronts of practical relevance, the maximum MPW amplitude depends almost exclusively on the steepness of the incident wavefront, regardless of its amplitude.

In a second paper, Wang et al. (2015b) extended the analysis to axisymmetric geometry and began to explore the influence of geometrical factors. In particular, the number and size of the openings along an exit region was varied. Only one wavefront was considered and, in common with the first paper, only one length of exit region was studied and its cross-section was identical to that of the tunnel. An important difference from the first tunnel was the adoption of an axisymmetric geometry. In general, this could be considered to be representative of a perforated extension tube on a pipe of circular cross-section. For the particular case of railway tunnels, however, it is more likely that attention will focus on the upper-half plane only. That is, the tunnel is of semi-circular cross-section and its horizontal diameter is coincident with the ground. This is broadly indicative of a tunnel emerging to open ground. The most important purpose of the paper was to explore the influence of the openings on the balance between MPWs radiated from the portal and MPWs radiated laterally from individual openings. The best balance was achieved with a total opening area roughly double the cross-sectional area of the tunnel. Unfortunately, this is much greater than the optimum area of well-designed entrance regions (typically less than half of the tunnel cross-sectional area - e.g. Vardy et al., 2015). That is, regions optimised for one of these purposes (entrance or exit) will also not be optimum for the other purpose. This result has clear implications for operators who wish to have the flexibility to use tunnels in either direction without loss of performance.

The previous papers consider only one exit region length and one cross-section, namely the same as that of the tunnel. As a consequence, they provide only a small fraction of the information available to designers of entrance regions through hundreds of papers that, collectively, have assessed a wide range of parameters. The present paper takes the next obvious step by assessing the influence of the most important outstanding parameters, namely the length and cross-sectional area of the perforated exit region. Future papers, possibly by the authors, but hopefully by others, could explore further configurations such as tapered regions and non-uniformly distributed openings. It will also be necessary to assess the generality of the predictions obtained for the specific configurations studied so far. For example, it is not safe to assume that the dependence of all reasonable exit regions on the properties of the incident wavefront will be similar to those obtained for the simple geometry assumed for the purposes of the first paper. Nevertheless, no attempt at such generalisation is made herein, although the development of an understanding of the influence of the lengths and cross-sectional areas of exit regions is intended as a step in that direction.

In common with most literature on MPWs, the focus herein is on the amplitudes of the emitted pressure disturbances even though the practical importance is also influenced by the associated frequency distributions (Hieke et al., 2011). This limitation is a practical necessity herein because the frequency 
distributions of MPWs are even more sensitive than their amplitudes to the particular shapes of the incident wavefronts. The latter depends on the particular origins of the wavefronts and on the characteristics of the particular tunnel. It would be wrong to limit this paper to the characteristics of any particular tunnel. Instead, the chosen incident wavefront is non-specific, but generically meaningful. For the practical convenience of readers as well as the authors, the chosen wavefront is identical to that used in the second paper (Wang et al., 2015b).

\subsection{Outline of the paper}

The main body of the paper gives detailed predictions of the behaviour of pressure waves propagating along perforated and unperforated exit regions and considers the consequences for MPW generation. First, however, attention is drawn in Section 2 to some detailed flow considerations that assist in the interpretation of the main predictions and Section 3 outlines and justifies the numerical method on which the predictions are based. The behaviour of waves propagating along unperforated exit regions is then described as a prelude to explaining propagation along perforated regions. The paper closes with a summary of the authors' views of the most important conclusions to be drawn from the paper. These are supplemented by some outcomes derived from the previous papers and discussed herein. In this way, the concluding remarks provide a concise overall summary of the main findings that have been obtained so far about the likely performance of perforated exit regions for tunnels.

\section{Important flow characteristics}

Before addressing the detailed influence of long exit regions on wave emissions, it is useful to consider some simple, easily understood phenomena that contribute to the overall behaviour. First, consider the case of a steep wavefront reflecting from the end of a duct with an infinite flange plate. The approaching wavefront will propagate in a plane-wave manner with uniform pressure over any particular cross-section. However, the subsequent pressure radiation beyond the duct will occur in all directions, not just axially. As a consequence, there must be a three-dimensional (3D) interaction between the internal and external flows. In typical one-dimensional (1D) analyses, this complication is disregarded and, instead, the pressure at the portal is prescribed to remain constant at all times (equal to the external atmospheric pressure). Account is taken of air flows out of the duct, but not of consequential local changes in the external pressure field. With this simplification, the predicted shapes of wavefronts reflecting back along the tunnel are the same as those of the incident wavefronts that give rise to them.

Figures 1(a) and 1(b) illustrate some 3D details of the reflection process. In Figure 1(a), a plane wavefront is shown approaching a tunnel portal and behaving in a plane-wave manner. After arriving at the portal (Figure 1(b)), it begins to expand laterally as well as axially. However, the freedom to expand laterally is not experienced simultaneously at all radii. It is felt first at the outer circumference of the tunnel and it becomes known at the inner radii only after expansion waves have spread outwards from the outer circumference of the portal. Until such waves arrive, the portion of the incident wave in the inner region will continue to propagate wholly axially. This is the cause of the reflection delays discussed above. Other features in Figure 1 are considered later in the paper.

Rudinger $(1955,1957)$ has shown that even in 1D analyses, it is possible to allow realistically for changes that occur during the reflection process and Brown and Vardy (1994) subsequently extended his analysis, taking advantage of greater computer power. It may be noted that the primary focus of these studies was on the consequences for internal reflections, whereas for MPW analyses, external changes are of more direct interest. Nevertheless, neither can be predicted accurately without simultaneously allowing for the other.

This behaviour is illustrated in Figure 2(a), which shows predicted cross-sectional average pressure histories at axial

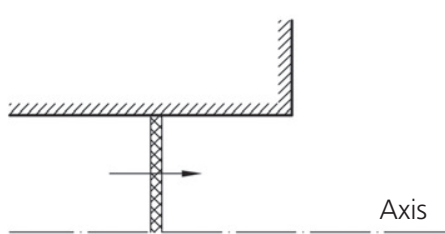

(a)

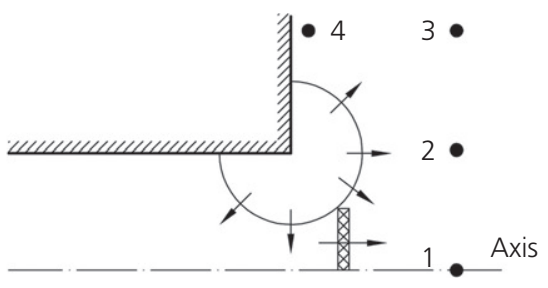

(b)

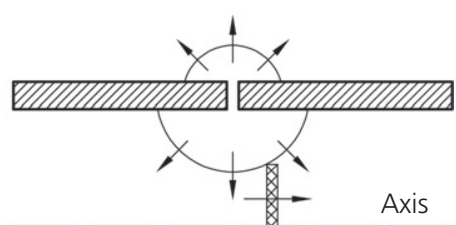

(c)

Figure 1. Indicative influence of 2D/3D geometry on plane-wave propagation: (a) plane wavefront approaching an exit portal; (b) reflection/transmission at an exit portal; and (c) reflection/ transmission at a wall opening 


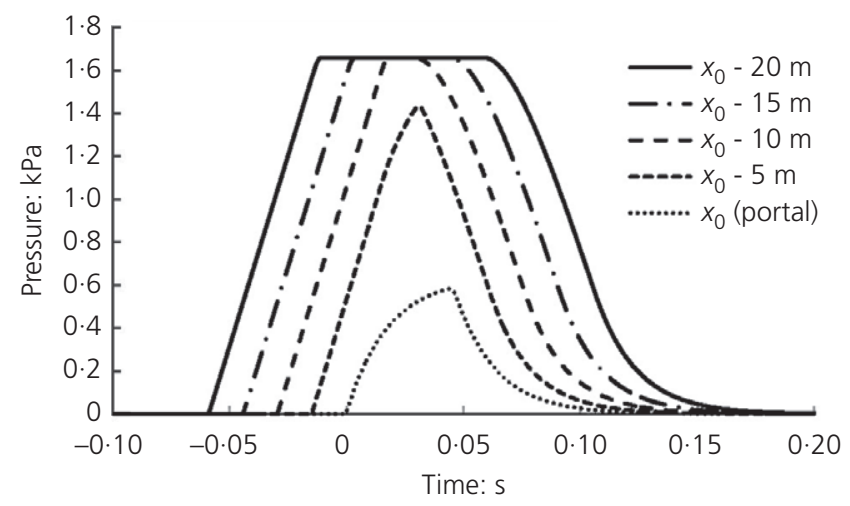

(a)

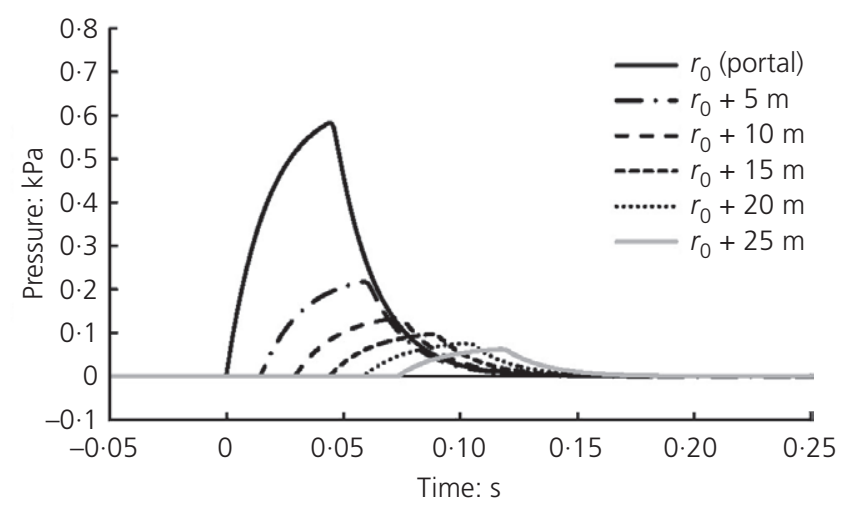

(b)

Figure 2. Radiation from a ramp wavefront reflecting at a flanged portal (note that different pressure scales are used in (a) and (b)):

(a) inside the tunnel and (b) beyond the portal

locations inside a tunnel with a flanged portal as a steepfronted ramp wavefront approaches and reflects at the portal. Figure 2(b) shows the corresponding pressure histories outside the tunnel as the resulting MPW expands spherically in the semi-infinite, hemispherical domain bounded by the portal flange plate. All of the histories are applicable on the tunnel axis and its extension beyond the portal. Inside the tunnel, the $x$-coordinate at the portal is $x=x_{0}$. Outside, the radial coordinate on the axis at the portal plane is $r=r_{0}$ and the origin of the spherical coordinates is on the tunnel axis, a distance $r_{0}$ upstream of the portal. Thus, the matching condition at the portal implies planar 1D behaviour inside the duct, but spherically radial 1D behaviour outside it. The change from planar to spherical geometry at the interface causes some inaccuracy, but the overall behaviour is realistic. The purpose of this particular figure is to illustrate the qualitative feature of pressure histories, so it is reasonable to use $1 \mathrm{D}$ analysis and to accept small quantitative inaccuracies. All subsequent figures are based on axisymmetric computational fluid dynamics (CFD) analysis.

By inspection, the pressure at any location along the inside of the tunnel increases to a maximum as the incident wavefront passes and then decays back to zero during reflection. At points sufficiently close to the portal, the first part of the reflected wavefront overlaps with the last part of the incident wavefront, so the maximum pressure is smaller than at locations further upstream. For the chosen incident wavefront, the initial increase is approximately linear, but the decay after the reflection is nevertheless strongly non-linear.

Outside the tunnel, the pressure amplitudes vary approximately inversely with distance from the portal (except very close to the portal). That is, the external conditions closely resemble those obtained for simple monopoles in standard acoustic analyses that underpin conventional methods of estimating MPWs outside tunnel portals.

The representation of the pressure field outside the tunnel by spherically radial $1 \mathrm{D}$ approximations is much more accurate than neglecting the pressure changes completely (as in typical 1D analyses of internal pressure waves). Nevertheless, it is only a first-order approximation to the true behaviour, which will inevitably exhibit a directional dependence, even in the limiting case of a semi-infinite external domain. This is illustrated in Figure 3, which shows pressure histories at points $1-4$ in Figure 1(b) when a steep ramp wave reflects at a simple portal. The details of the tunnel and the incident wavefront are not

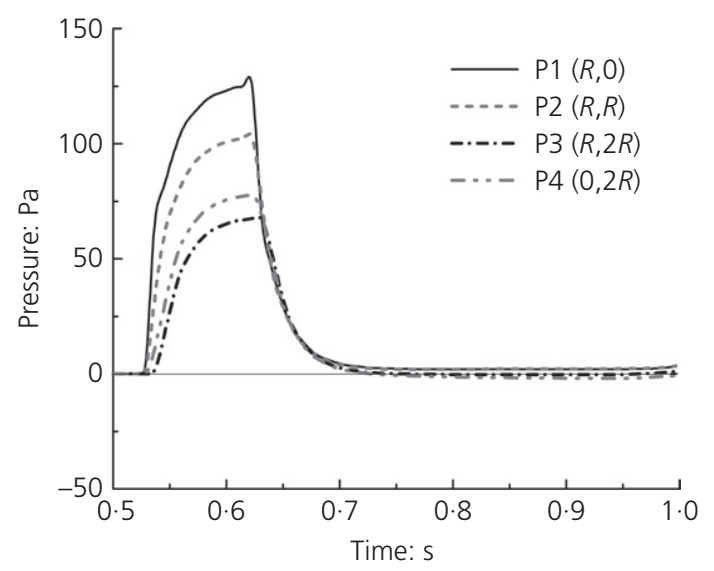

Figure 3. Pressure histories at locations just outside a simple portal (the points 1-4 are defined in Figure 1(b)) 
important for the qualitative purposes of this figure, but note that points $1-4$ are equally spaced.

Figure 1(c) illustrates a related local effect, namely the disturbance caused by an opening in the side of a duct along which a wavefront is propagating. This is an especially important event for the purposes of the rest of this paper. From a planar 1D standpoint, the opening causes a partial reflection of the wavefront and reduces the pressure amplitude of the wavefront that continues further along the tunnel. Simultaneously, the consequential mass flow through the opening causes a disturbance to propagate into the external atmosphere. In practice, inside the tunnel, the opening first influences the outer region of flow and finite time must elapse before it can influence the whole cross-section. One important consequence of this is that the internal wavefront ceases to propagate in a planar 1D manner, at least until the wavefront has moved a significant distance beyond the opening. In this paper, it always encounters further openings before this stage is reached - except for waves reflecting back upstream into a region of tunnel with no openings.

The extent of the deviation from plane-wave behaviour will depend on the size of the opening relative to the duct area and also on its proximity to other causes of non-uniform behaviour (e.g. other openings or the duct portal). In principle, however, it is potentially important in the context of tunnel design because it shows that 1D methods of analysis will necessarily fail to predict potentially significant effects. This is a rare example where it is not safe to rely on the usual assumption that 1D methods are adequate for the modelling of wave propagation inside tunnels. Wang et al. (2015a) presented detailed comparisons of alternative predictions based on 1D and 2D analyses for a tunnel exit region with multiple openings. It was shown that $1 \mathrm{D}$ analyses can predict reflected wavefronts upstream of perforated regions with sufficient accuracy for practical design purposes, but that the corresponding predictions of wavefront propagation along the perforated regions themselves are significantly less accurate. One reason for these different outcomes is that the dominant (early) part of the reflected wavefront has been influenced by only a few openings, whereas the pressures radiated from the tunnel portal arise after the incident wavefront has been influenced by all of the openings along the region.

\section{Methodology}

For the rest of this paper, attention is focused on predictions obtained using a 2D axisymmetric analysis. This enables the main characteristics of the $3 \mathrm{D}$ phenomena to be captured without incurring the large central processing unit (CPU) penalty that would obtain if a fully $3 \mathrm{D}$ analysis were used. Furthermore, even if a fully 3D analysis were used, it would be premature to consider strongly $3 \mathrm{D}$ geometries before acquiring an adequate understanding of more simple geometries.

A planar 2D version of the theoretical method used in the following simulations has been described in detail by Wang et al. (2015a). The only significant difference in the analysis used herein is the use of cylindrical polar coordinates instead of 2D Cartesian coordinates. It would not be appropriate to reproduce the full methodology herein, but, for completeness, the main features are described in Appendix 1 together with the methodology followed in ensuring that the predictions are free from significant numerical error.

The geometrical configuration is depicted in Figure 4. A uniform-bore tunnel of radius $R_{\mathrm{tun}}=5 \mathrm{~m}$ has a uniform-bore perforated exit region with uniformly distributed openings in its wall. In some simulations, the radii of the tunnel and the exit region are equal. In others, the radius of the exit region exceeds that of the tunnel. Only the end portion of the tunnel is simulated and the upstream pressure is prescribed to increase linearly from zero to a maximum of $2 \mathrm{kPa}$ in a period of $0.05 \mathrm{~s}$. This causes a steep-fronted wave to propagate along the tunnel towards the perforated exit region. Some artistic licence has been taken in the representation of the openings in the cross-sectional figure. In a literal interpretation of axisymmetric geometry, the openings shown in the longitudinal section
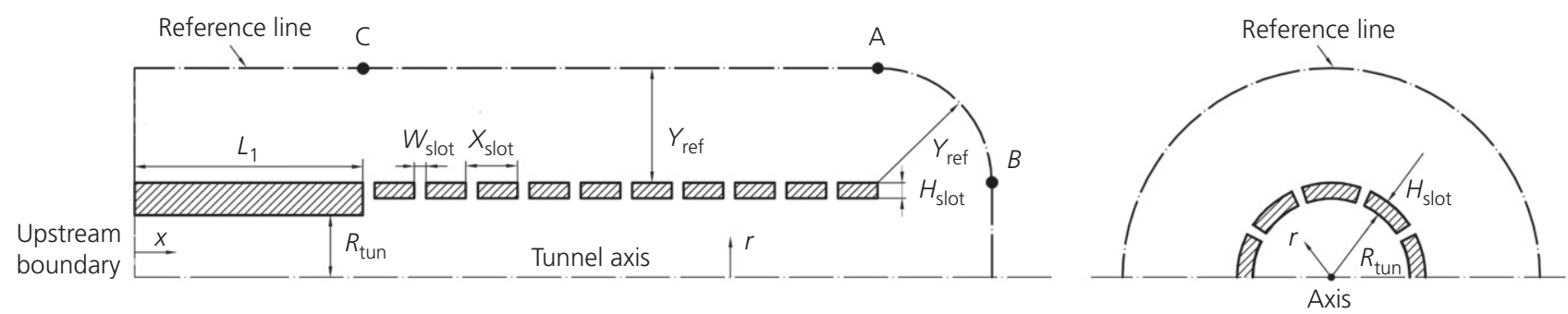

Figure 4. Tunnel geometry and reference line (the chosen reference line is $25 \mathrm{~m}$ from the outer surface of the tunnel) 
necessarily extend around the whole of the circumference. This cannot be shown in the cross-section and therefore a possible practical configuration is shown instead. For the purposes of this paper, the exact local distribution of openings is far less important than their overall distribution along the region.

Figure 4 includes a reference line at a uniform distance of $Y_{\text {ref }}=25 \mathrm{~m}$ from the tunnel surface and from the portal plane. This line is used below to illustrate how pressure variations outside the tunnel vary with position. In the case of simple tunnel portals, attention in the open literature commonly focuses on pressures around 20 or $25 \mathrm{~m}$ from a tunnel portal. The use of standard reference locations such as these assists in interpreting the outcomes for various exit configurations and in comparing outcomes for different tunnels. In the case of perforated exit regions, however, it is not sufficient to focus exclusively on conditions beyond the exit portal because MPWs can also be generated from individual openings. The reference line is a device for allowing for the greatly increased region of interest in a simple, but informative manner.

A big simplification is achieved by restricting the simulations to the idealised case of inviscid flow. This is a good approximation to wave propagation in compact regions such as those considered herein even though it would not be a good approximation for the purpose of long-term, steady flow simulations.

\section{Exit regions with no openings}

To provide a basis for comparisons, Figure 5 shows pressure histories as the prescribed steep-fronted wavefront approaches and reflects at the exit portal of a simple tunnel with an unperforated exit region. Figure 5(a) shows instantaneous pressure profiles on the tunnel axis (i.e. $r=0$ ) up to the time when the reflection process begins $(\sim 0 \cdot 75 \mathrm{~s})$. The wavefront remains almost unchanged during this period except for a slight increase in its steepness.

Figure 5(b) shows the corresponding profiles at later times that is, after the reflection process has begun. The shape of the reflected wavefront inside the tunnel exhibits the characteristic delays deduced by Rudinger $(1955,1957)$. Beyond the portal, the pressure amplitudes are much smaller than that of the incident wavefront. At the portal itself, the pressure is much closer to the initial external pressure than to the amplitude of the incident wavefront except for a very short period discussed above using Figure 1(b). This is the justification for the usual simplification described above for 1D simulations of internal pressure disturbances.

For completeness, Figure 5(c) shows successive profiles along the reference line, illustrating the spreading of the external pressure field away from the portal - in both negative and positive

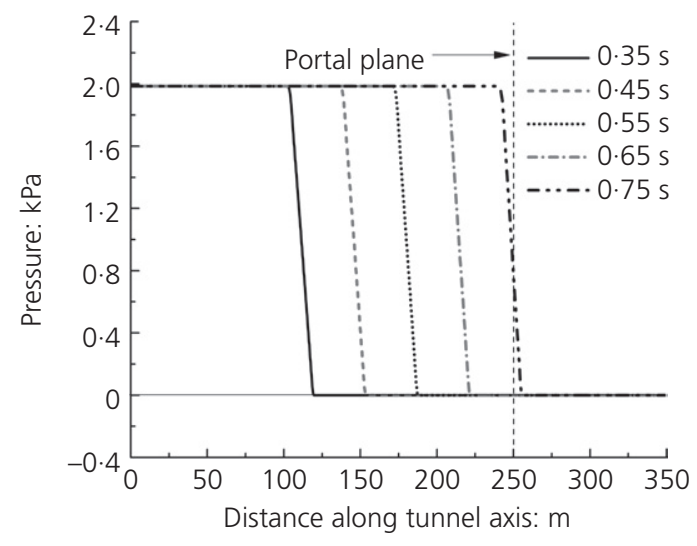

(a)

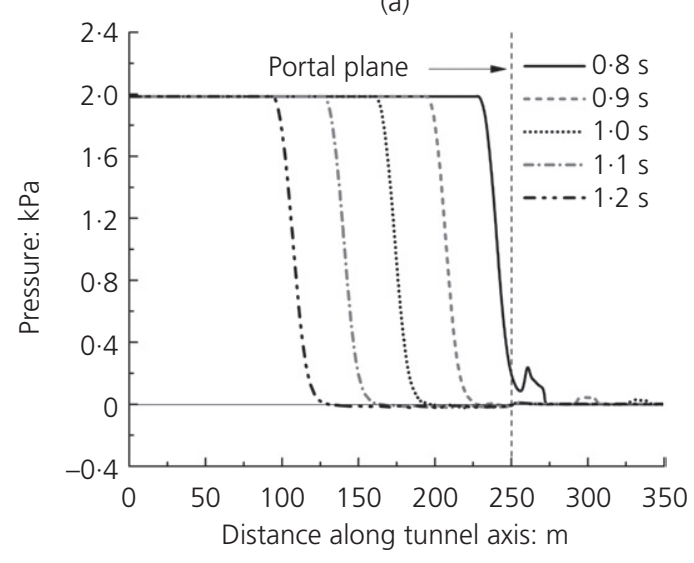

(b)

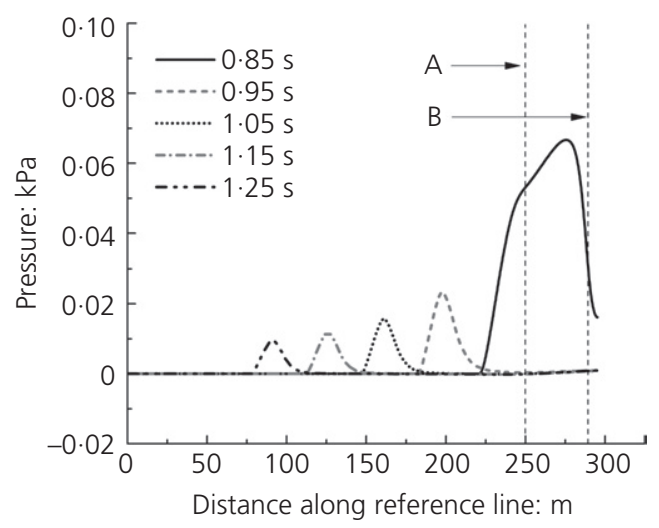

(c)

Figure 5. Pressure profiles along the tunnel axis and reference line: unperforated exit region $\left(R_{\mathrm{tun}}=5 \mathrm{~m}\right.$ ) (the points $\mathrm{A}$ and $\mathrm{B}$ are defined in Figure 4): (a) wavefront approach; (b) reflection at the portal; and (c) pressure along the reference line

$x$-directions. The amplitudes decrease with increasing distance because the pressure wave expands as it propagates into the $3 \mathrm{D}$ space. An increased pressure scale is used in this figure because the pressures are much smaller than those inside the tunnel. 


\subsection{Influence of cross-sectional area of exit region}

Figure 6 compares predictions for the same case with corresponding predictions for a tunnel with an exit region of

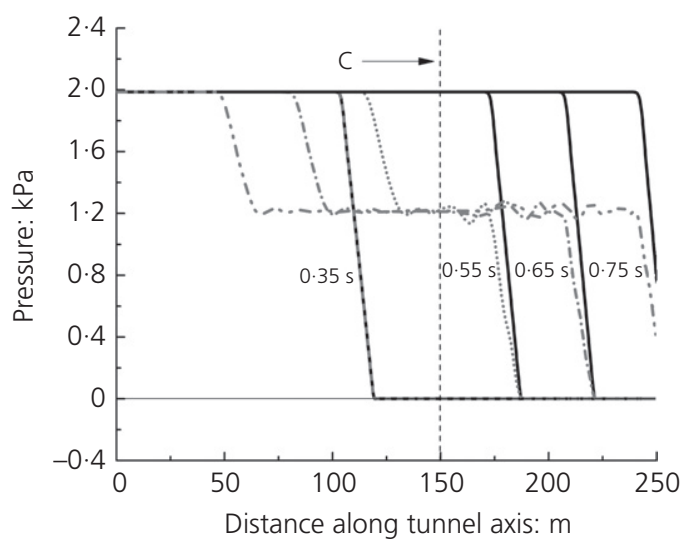

(a)

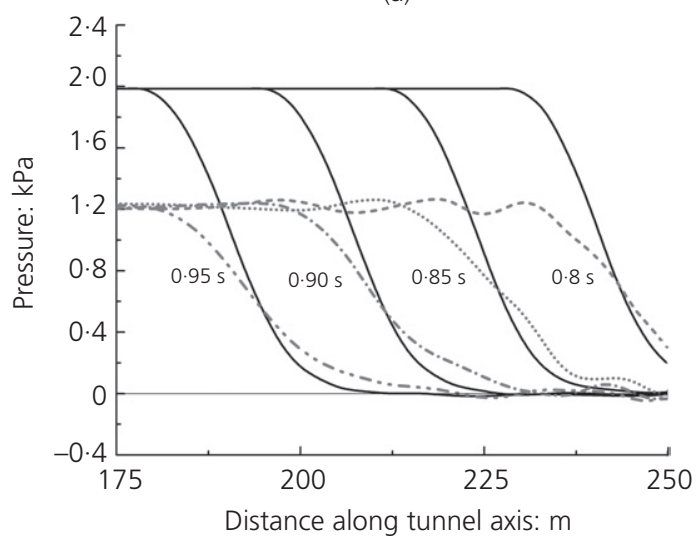

(b)

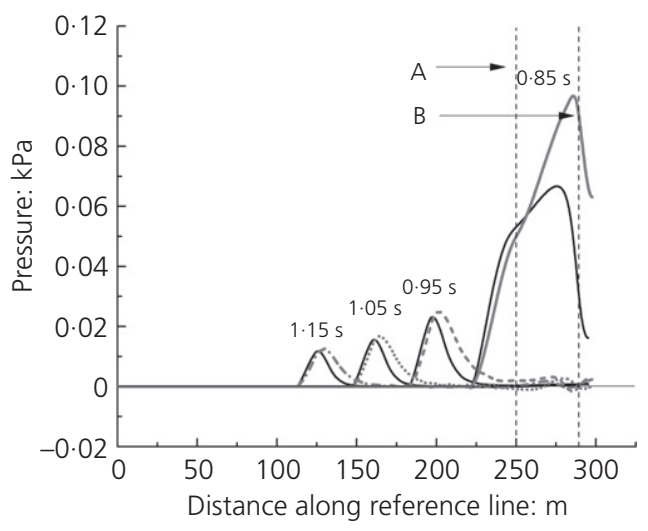

(c)

Figure 6. Pressure profiles along the tunnel axis and reference line: unperforated exit region with enlarged area (continuous lines: $R_{\mathrm{ext}}=R_{\text {tun }}=5.0 \mathrm{~m}$, broken lines: $R_{\mathrm{ext}}=7.5 \mathrm{~m}$ ) (points $\mathrm{A}, \mathrm{B}$ and $C$ are defined in Figure 4 ; point $C$ is at the end of the main tunnel) increased area (i.e. as Figure 4, except without the openings). Before reaching the step change in area (e.g. $t=0.35 \mathrm{~s}$ ), the two cases are identical. After reaching the step, however, the pressure reduces strongly, reducing the pressure amplitude of the onward-moving wavefront and causing a strong negative wavefront to propagate back upstream. The negative reflected wavefront causes the velocity upstream of the step to increase. In contrast, the velocity downstream of the step is smaller than in the case with no increase in area. This is a consequence of the need for mass-flow continuity at the step. The net result is a decrease in pressure and velocity amplitudes of the onwardmoving wavefront, but an increase in the associated mass-flow rate in comparison with the rate before the wavefront reached the step.

Figure 6(c) shows that when the wavefront reaches the portal, it causes a greater MPW amplitude than that for a tunnel with no area-step even though the step reduces the pressure amplitude of the wavefront reaching the portal. This is because the critical property of an incident wavefront for MPW generation is the rate of change of mass flow that it induces, not the corresponding rates of change of velocity or pressure. Figure 7 shows that the rate of change of mass-flow rate through the portal increases with the cross-sectional size of the exit region (Note: Anyone who is surprised that increasing the exit region area has the consequence of increasing the MPW even though it reduces the amplitude of the pressure wave arriving at the portal should consider the shapes of musical instruments such as trumpets and oboes).

\subsection{Influence of length of exit region}

The length of an enlarged-area (unperforated) exit region is much less important than its cross-sectional area. Indeed, provided that the step change in area is not great, the strongest influence of the length is on the intervals between the key

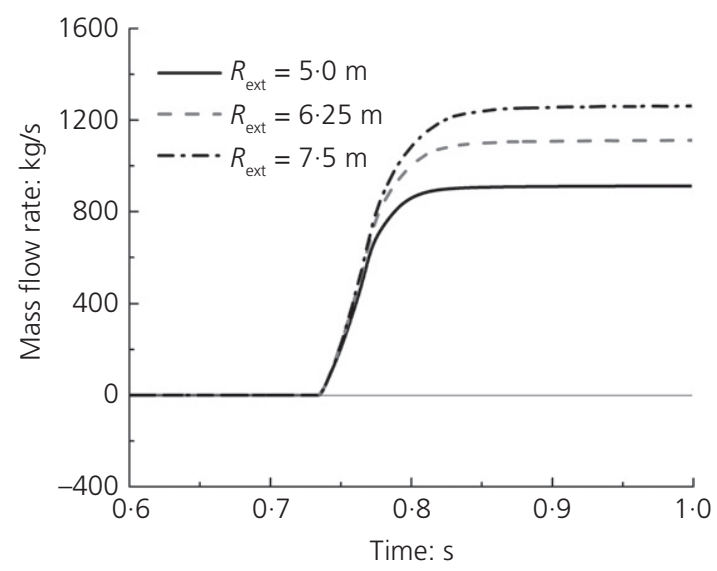

Figure 7. Mass-flow rate through the exit portal plane $\left(R_{\text {tun }}=5 \mathrm{~m}\right)$ 
events observable in Figure 6. This, in turn, is important for MPW purposes only if the intervals are so short as to be comparable with the time needed for pressures to equalise over a cross-section - that is, if the length of the region is no more than one or two tunnel diameters. Such short regions are unlikely to be advantageous in the case of railway tunnels even though they could be relevant in other applications where high-frequency phenomena are dominant. Accordingly, for conciseness, no predictions are presented herein to illustrate the length dependence of unperforated exit regions (but see Section 5.2 for the length dependence of perforated exit regions).

\section{Exit regions with openings}

Figure 8(a) shows successive pressure distributions as a wavefront approaches and travels along a $100 \mathrm{~m}$ long perforated extension region with the same cross-sectional dimensions as

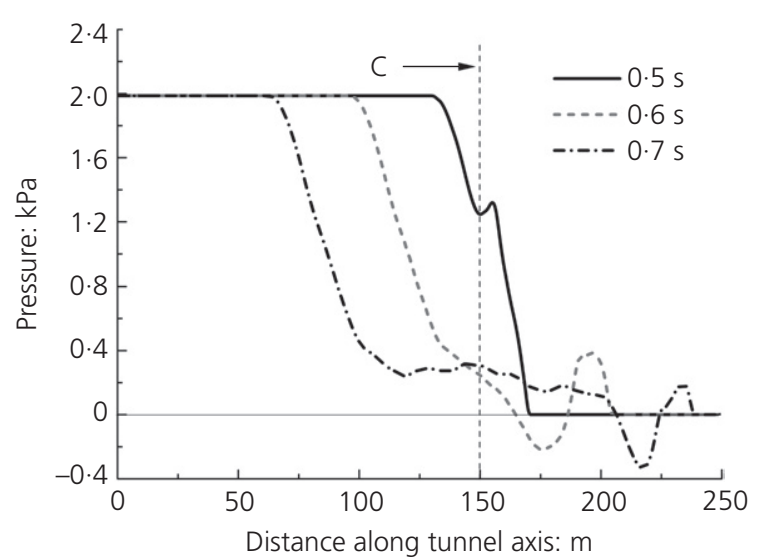

(a)

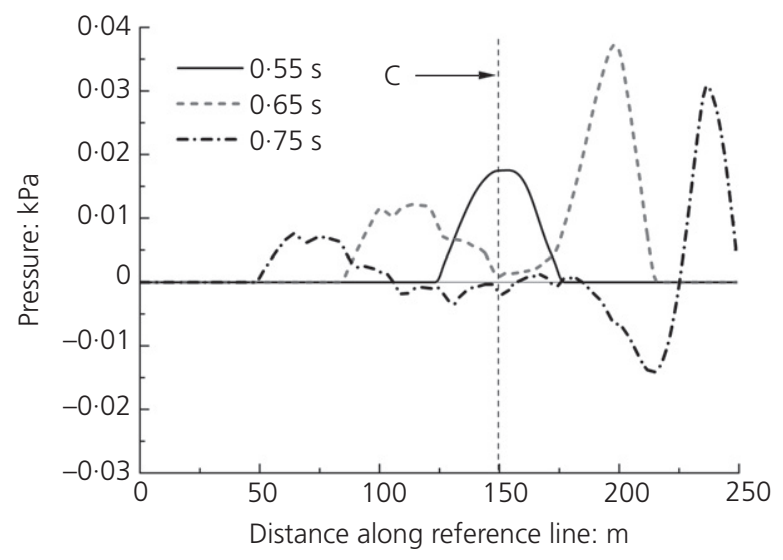

(c)

Figure 8. Pressure profiles along tunnel axis and reference line: perforated exit region $\left(R_{\text {tun }}=5 \mathrm{~m}\right.$ ) ( points $\mathrm{A}, \mathrm{B}$ and $\mathrm{C}$ are defined in Figure 4; point $C$ is at the end of the main tunnel): (a) at $r=0$, as the wavefront approaches the portal plane; (b) at $r=0$, after the the tunnel (i.e. $R_{\mathrm{ext}}=R_{\text {tun }}=5 \mathrm{~m}$ ). The region has ten openings at intervals of $2 R_{\text {tun }}=10 \mathrm{~m}$. Each is $0.5 \mathrm{~m}$ wide (axially), so their total area is $5 \%$ of the surface area of the perforated region or twice its cross-sectional area. The pressure distributions for this case differ greatly from those shown in Figure 5 for an exit region with no openings. This is evident soon after the wavefront reaches the first opening (at $x=150 \mathrm{~m}$ ). Each opening reduces the amplitude of the leading part of the wavefront inside the tunnel and the cumulative influence of all the openings is a major reduction in the amplitude of the wavefront arriving at the portal. The consequential reduction in rates of change of mass-flow rate causes a big reduction in the MPW radiated from the exit portal (Figure 8(b)).

There is a price to be paid for this reduction, however, namely the existence of pressure disturbances emitted from the openings themselves (Figure 8(c)). In this particular example, the

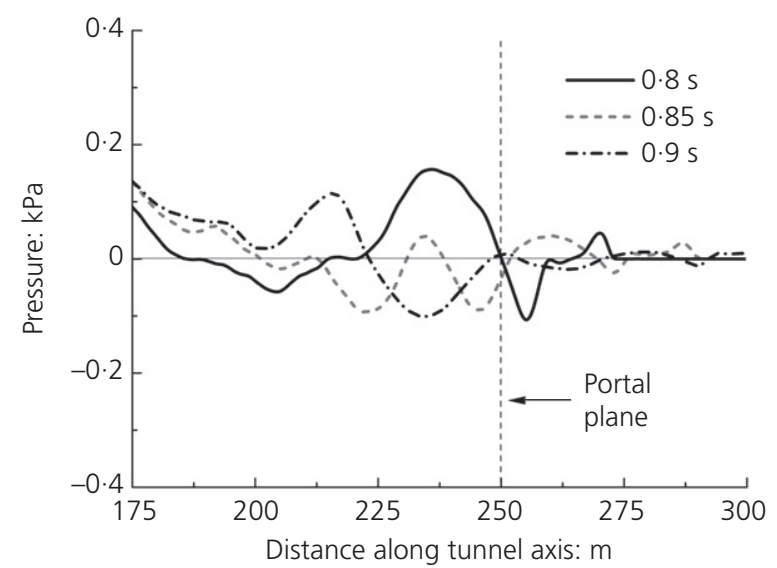

(b)

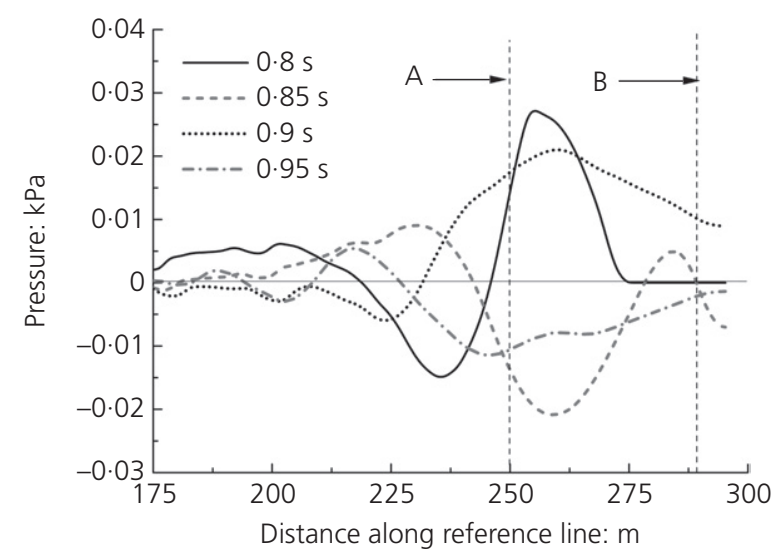

(d)

wavefront toe reaches the portal plane; (c) along the reference line, as the wavefront approaches the portal plane; and (d) along the reference line, after the wavefront toe reaches the portal plane 
greatest induced pressures along the reference line upstream of the portal plane (i.e. within $0<x<250 \mathrm{~m}$ ) occur before emissions begin through the portal itself. The combined influence of disturbances through the portal and through the side openings can be seen from Figure $8(\mathrm{~d})$. In this instance, the largest pressures on the reference line beyond the portal are broadly comparable with the largest ones alongside the tunnel (Figure 8(c)).

Other differences exist between the conditions obtained in exit regions with and without openings. One of these is the existence of negative wave pressures both inside and outside the tunnel as the wavefront propagates towards the exit. In part, these are a consequence of wave focusing as waves from the tunnel circumference converge towards the axis and then diverge as they reflect back towards the circumference

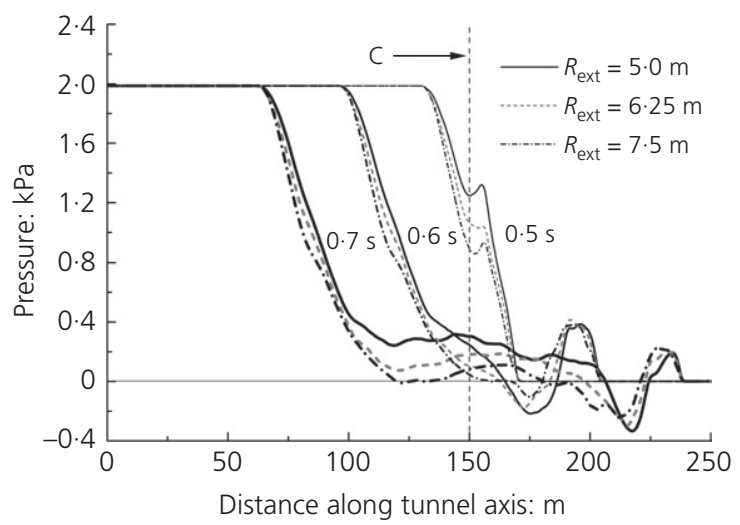

(a)

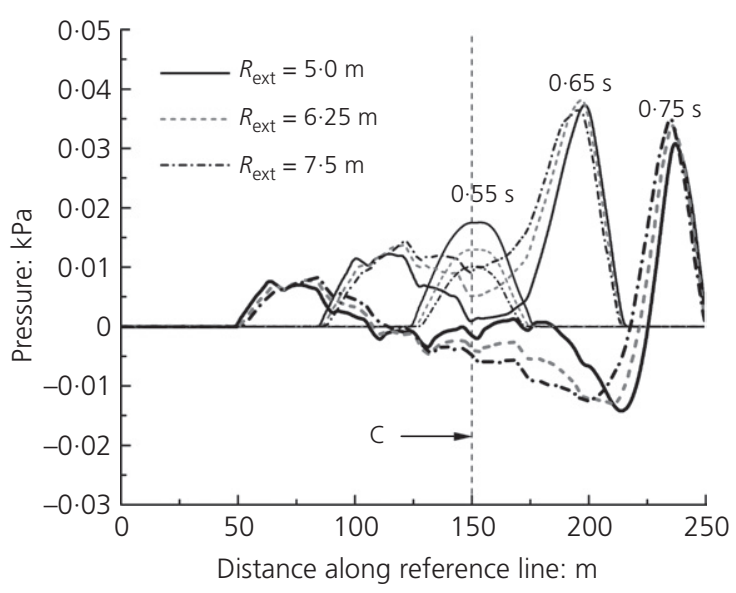

(c)

Figure 9. Pressure profiles along the tunnel axis and reference line: influence of cross-sectional area of perforated exit region $\left(R_{\text {tun }}=5 \mathrm{~m}\right)$ : (a) at $r=0$, as the wavefront approaches the portal plane; (b) at $r=0$, after the wavefront toe reaches the portal
(Figure 1(c)). In part, they are a consequence of superpositions of multiple wavefronts resulting from interactions between reflections from the various openings. The detailed outcome in any particular instance depends on the particular configuration of openings, but the existence of negative values is characteristic of perforated exit regions in general when the openings are sufficiently large.

\subsection{Influence of cross-sectional area of exit region}

Figure 9 shows predicted pressure fluctuations for tunnels with $100 \mathrm{~m}$ long perforated exit regions of increased area. Predictions for regions with inner radii of 6.25 and $7.5 \mathrm{~m}$ are compared with predictions for the base case with the same radius as the tunnel - that is, $5 \mathrm{~m}$. The axial width of the openings is the same for all cases (i.e. $0.5 \mathrm{~m}$ ); therefore the total area of the openings is the same proportion as the exit region

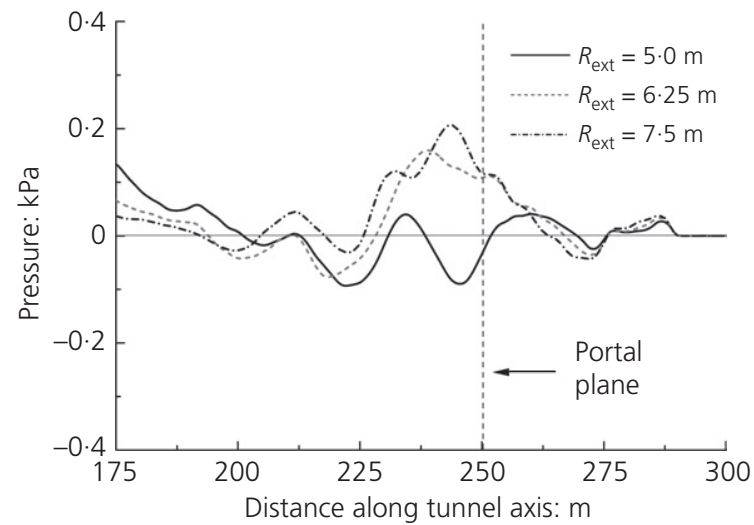

(b)

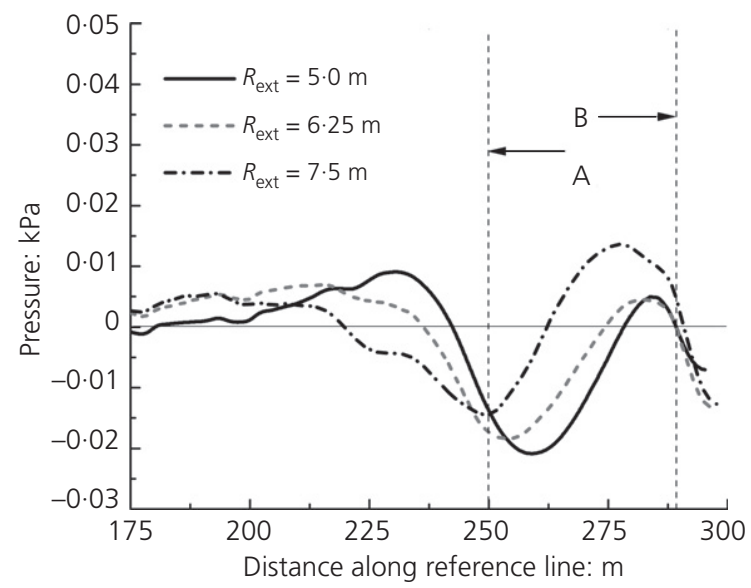

(d)

plane; (c) along the reference line, as the wavefront approaches the portal plane; and (d) along the reference line, after the wavefront toe reaches the portal plane 
surface area in all cases. For the large-radius extensions, it follows that the total area of the openings is a decreased proportion of the local cross-sectional area, but an increased proportion of the cross-sectional area of the main tunnel.

In all cases, the wall thickness is chosen as $1 \mathrm{~m}$, so the outer radius of the exit regions increases in line with the increase in the inner radius. The outer radius of the main tunnel is maintained equal to that of the exit region to avoid the need for a step in the outer profile. Such a step could be simulated with ease, but it is avoided herein because it would complicate the interpretation of pressures alongside the tunnel. Even with this simple geometry, a compromise is necessary in the choice of a suitable reference line. The most obvious choices are $(a)$ using the same absolute location for all cases or $(b)$ having the same distance between the reference line and the tunnel wall in all cases. These two possibilities are mutually exclusive. The latter has been chosen herein, partly because it is consistent with choosing the same reference line location beyond the portal plane in all cases considered in this paper.

Figure 9(a) shows pressure distributions along the $x$-axis as an incident wavefront approaches the tunnel portal. It can be seen that the distributions with enlarged, perforated regions approximate more closely to those with unenlarged, perforated regions (Figure 8) than to those with enlarged, unperforated regions (Figure 6). That is, the principal cause of the amplitude reductions inside the tunnel in these examples is the flow through the side openings, not the increased cross-sectional area. Figure 9(b) shows pressure distributions on the axis at a later instant $(t=0.85 \mathrm{~s})$ after the initial development of the MPW beyond the portal. At this particular instant, the amplitudes for the enlarged regions exceed those for the unenlarged

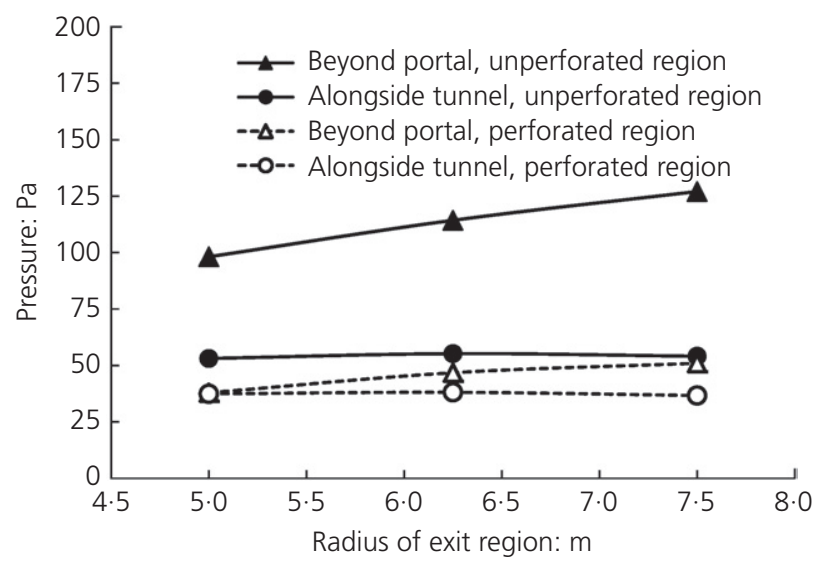

Figure 10. Overall maximum pressures along the reference line: influence of the cross-sectional area of the perforated exit region $\left(R_{\text {tun }}=5 \mathrm{~m}\right)$ region, but this is not a universal outcome (see the later discussion in Figure 10).

Figures 9(c) and 9(d) show corresponding pressure distributions along the reference lines which, although equidistant from the outer surfaces of the tunnels, are at different absolute radii in the three cases. The particular instants at which the graphs are shown have been chosen to highlight the principal features of the behaviour. Alongside the tunnel (Figure 9(c)), the outcome does not depend strongly on the cross-sectional area. Beyond the portal (Figure 9(d)), the apparent dependence is greater, but note that the figure is applicable only for the particular size and distribution of the chosen openings in the wall and only at the particular instant depicted.

For practical design purposes, instantaneous conditions are much less useful than the overall maxima. Accordingly, Figure 10 shows predicted maximum pressures on the reference line alongside and beyond the exit region for each case considered above. The continuous lines with filled symbols show maximum values for unperforated exit regions and the broken lines with open symbols show the corresponding values for the perforated regions. For both types, the predicted maximum alongside the exit region is almost independent of the crosssectional size. Likewise, for both types, the corresponding maximum beyond the portal increases with the cross-sectional size, but much less strongly for perforated regions than for unperforated regions. However, it can be inferred that the opening sizes are not optimum for the larger radius exit regions. With slightly wider openings, the maximum pressures alongside the tunnel and beyond the portal would be equalised at intermediate values between those in Figure 10. This implies a weaker dependence on the cross-sectional size than that found in Section 4.1 for unperforated regions.

\subsection{Influence of length of exit region}

Figure 11 shows predicted pressures for four exit regions with identical cross-sectional sizes (equal to the main tunnel), but different lengths, namely 50, 100, 150 and $200 \mathrm{~m}$, respectively. It is not expected that very long exit regions will be constructed in the foreseeable future, but this choice of lengths assists in clarifying behaviour. Each exit region has ten slots with widths of $0.5 \mathrm{~m}$, so the total area of openings is the same in all four cases, namely twice the tunnel cross-sectional area. However, the distance between successive openings is proportional to the overall length, so the area density of the openings as a proportion of the wall area varies inversely with length of the region. The vertical broken lines labelled A and B correspond to points A and B in Figure 4 and the broken line $\mathrm{C}$ indicates the beginning of the exit region (i.e. the location of the first side opening).

As the regions have different lengths, comparisons of individual pressure distributions would be less meaningful than 


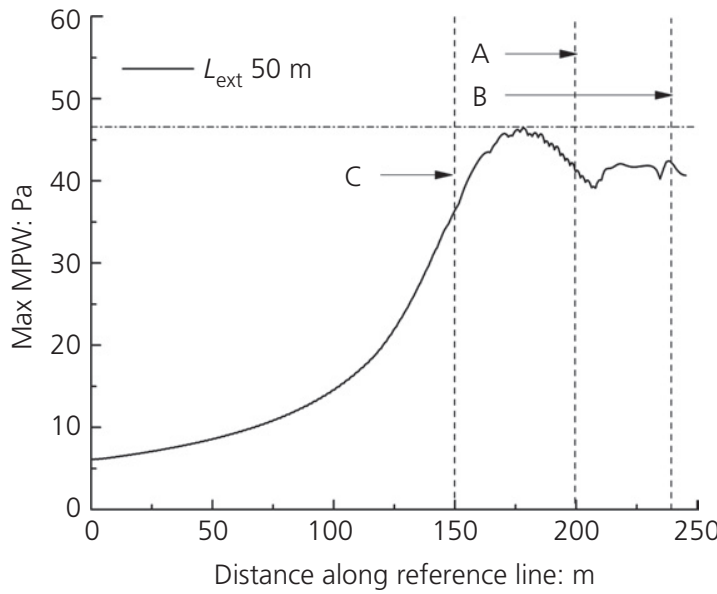

(a)

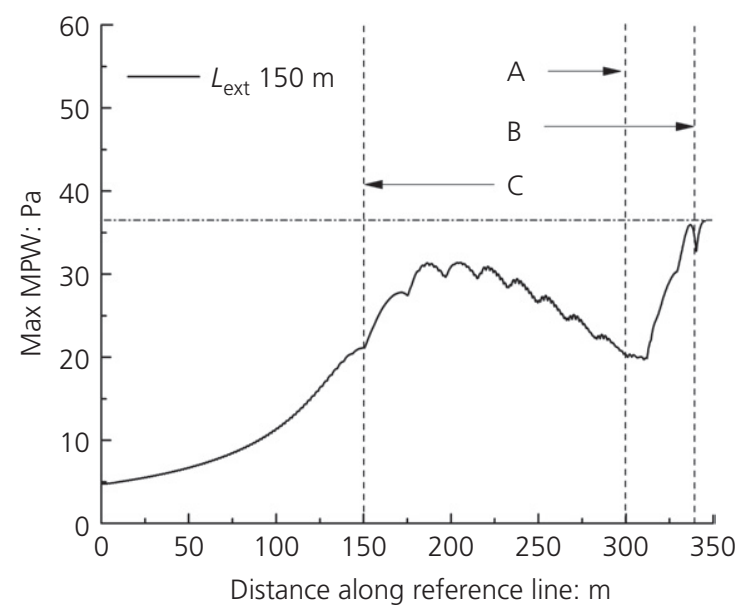

(c)

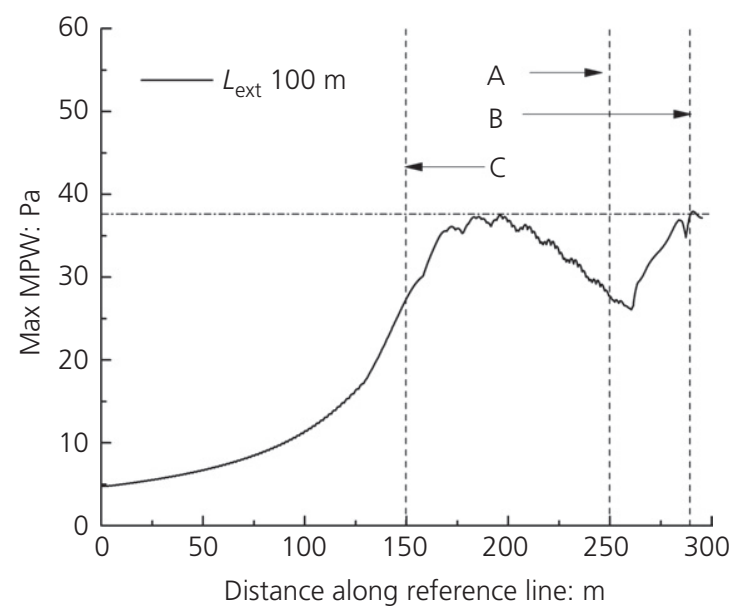

(b)

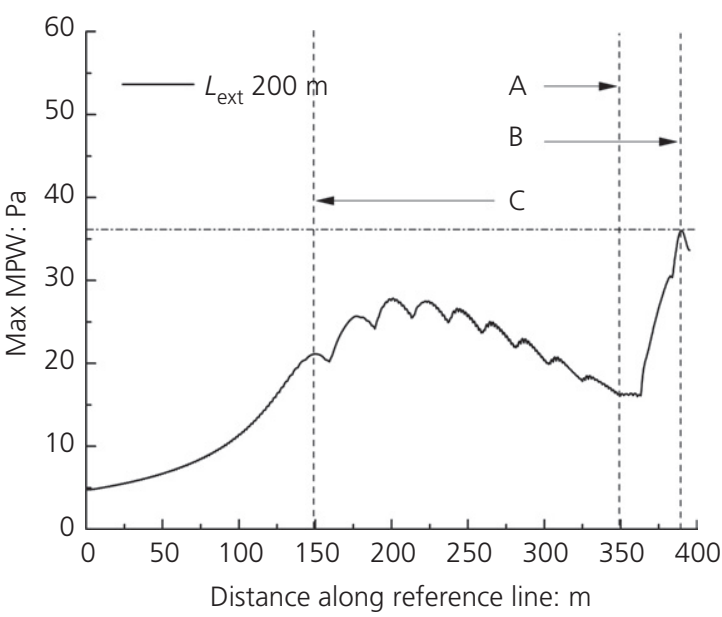

(d)

Figure 11. Maximum pressures along the reference line: influence of the length of the perforated exit region $\left(R_{\text {ext }}=R_{\text {tun }}=5 \mathrm{~m}\right):\left(\right.$ a) $L_{\text {ext }}=50 \mathrm{~m}$; (b) $L_{\text {ext }}=100 \mathrm{~m}$; (c) $L_{\text {ext }}=150 \mathrm{~m}$; and (d) $L_{\text {ext }}=200 \mathrm{~m}$

comparisons presented above for other configurations. Accordingly, instead of presenting instantaneous pressures, Figure 11 shows maximum pressures at each location along the reference line. The time at which the maximum value occurs depends on the particular location, but the timing is of little practical importance and so this method of comparison is instructive. Moreover, it has the spin-off advantage of enabling attention to be drawn to a factor that has received little attention above, namely the relative amplitudes of MPWs beyond the portal and alongside the perforated region. The horizontal broken lines in the figures assist in this purpose.

Figure 11(b) corresponds to the case considered above in Figure 8. It shows that, alongside the tunnel, greater maximum pressures occur in the central part of the exit region than at either end of it. This is consistent with the results obtained by Fukuda et al. (2013) in a numerical and experimental study of pressures alongside snow sheds in Japanese tunnels. The figure also shows that the greatest maxima beyond the portal (i.e. to the right of line $\mathrm{A}$ in the figure) occur close to the tunnel axis $(r=0)$. The dimensions of the openings are such that the maxima in these two regions are approximately equal.

Now consider Figures 11(a), 11(c) and 11(d). In each of these, the maximum value alongside the tunnel differs from the maximum beyond the portal, thereby demonstrating that the optimum size of the openings depends on the length of the exit 


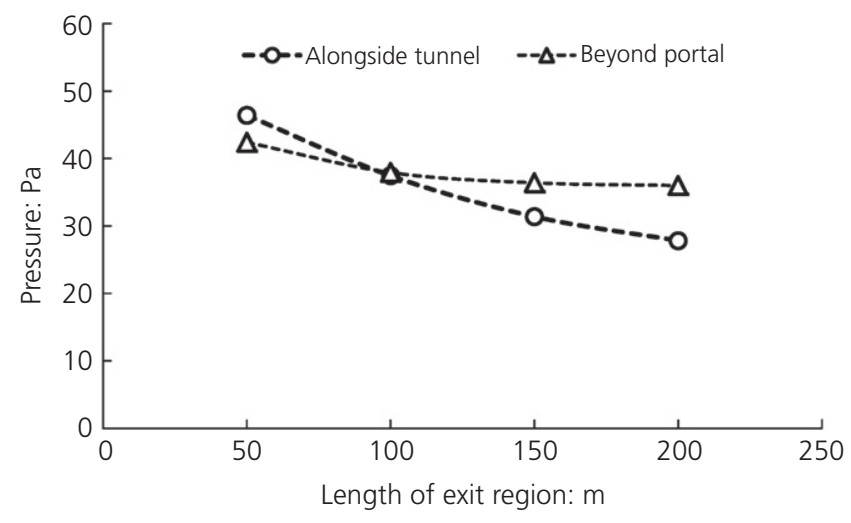

Figure 12. Overall maximum pressures along the reference line: influence of the length of the perforated exit region $\left(R_{\text {ext }}=R_{\text {tun }}=5 \mathrm{~m}\right)$

region. It can be inferred from Figure 11(a) that, for a shorter region, somewhat smaller opening areas would be advantageous; this would reduce emissions from the openings and increase emissions from the portal. Similarly, Figures 11(c) and 11(d) show that larger areas would be advantageous for longer exit regions.

These trends are presented in a concise visual form in Figure 12. The continuous line shows maximum values along the reference line beyond the portal for each of the four region lengths and the broken line shows corresponding maxima alongside the tunnel. In both regions, the predicted maximum pressures decrease with increasing region length. It can be inferred that the chosen size of the openings is close to optimum for the $100 \mathrm{~m}$ region, but that somewhat smaller openings would be preferable for the shorter region and larger openings would be preferable for longer regions. The optimum condition with equalised values in the two regions would lie between the two curves in Figure 12, thereby confirming that their potential effectiveness increases with increasing length.

\section{Concluding comments}

The potential effectiveness of long, perforated exit regions for reducing the amplitudes of MPWs emitted from railway tunnels has been investigated. The key outcomes of previous work have been summarised and have been extended to include the influence of the length and cross-sectional area of the regions. The regions can greatly reduce MPWs radiated from tunnel portals, but only at the expense of increasing MPWs alongside tunnels. In any particular application, the optimum size of openings will depend on the acceptability of MPWs in both these locations.
Previous work has shown that the effectiveness of perforated exit regions depends on the amplitude as well as the steepness of the wavefront arriving at the exit region. Moreover, using the same indicative wavefront as herein, an optimum distribution of equal-size openings in a $100 \mathrm{~m}$ long exit region with the same cross-sectional area as the tunnel has been found. In this paper, it has been further shown that the optimum area of the openings depends on both the length of the exit region and its cross-sectional size. The potential effectiveness of the regions increases with increasing length and decreases with increasing area. However, the latter effect is much smaller than with unperforated exit regions, so it does not constitute a major constraint on the choices open to designers. In particular, modest increases in cross-sectional area will be acceptable if they are convenient for construction purposes.

Perforated exit regions would be especially useful when the use of long entrance regions would not be expected to be sufficiently effective. The two types of tunnel extension have a close family resemblance, but the optimum opening areas identified herein for exit regions are considerably greater than the optimum areas for entrance regions. This is an inconvenient result for operators wishing to design tunnels that are equally effective for trains travelling in either direction. Indeed, some tunnels already have 'entrance' regions at both ends.

Although this paper provides strong guidance on the behaviour of perforated exit regions, it is not intended as a comprehensive design guide. There are far too many variables to avoid the need for site-specific design in any particular application. However, the general methodology will be suitable for many applications and, since it is founded on the use of a common form of readily available software, it can be regarded as a practical design method. If required, however, it would be simple to extend the method to embrace fully $3 \mathrm{D}$ geometries that enable special attention to be paid to specific locations outside a tunnel. However, that would greatly restrict the number of alternative designs that could be studied rapidly so it is preferable to use the simpler axisymmetric approach for initial scoping studies.

\section{Acknowledgements}

The authors are grateful to the following bodies that provided financial support for the project: (a) China Scholarship Council, (b) National Natural Science Foundation of China (grant no. U1334201 and (c) UK Engineering and Physical Sciences Research Council (grant no. EP/G069441/1).

\section{Appendix 1: Theoretical basis}

The theoretical method used herein is similar to that presented by Wang et al. (2015a), the only important differences being the use of polar coordinates and axisymmetric geometry 
instead of Cartesian coordinates and planar 2D geometry. The methodology is reproduced here in a summarised form for information, but the equations are not reproduced.

The simulation domain is shown in Figure 13. It includes the end region of the main tunnel, the perforated exit region and the external flow field (which, for clarity, is shown as much smaller than the true computational domain). In the upstream boundary of the tunnel region, the pressure is prescribed to increase linearly from the ambient pressure to a predetermined value at which it is then held constant. This causes a rampfronted wave to propagate along the tunnel. Each of the other boundaries is one of

a solid surface at which free-slip conditions are assumed

- the tunnel axis (coincident with the coordinate axis)

a boundary through which waves can pass without strong reflections and which is sufficiently far from the tunnel to have negligible influence during the period simulated.

Initially, the pressure and (zero) velocity are uniform over the whole domain. All subsequent variations are consequences of the prescribed pressure variation at the upstream tunnel boundary.

A highly desirable feature of the theoretical method is that it can be implemented using any of a range of software packages that are widely available. The particular software used herein is ANSYS FLUENT [26] (ANSYS, Inc, 2011), with the following options: density-based solver, Roe-FDS Flux Scheme, third-order MUSCL for spatial discretisation and second-order implicit time stepping for temporal discretisation.

Numerous advanced numerical schemes for simulating wave propagation have been proposed in the literature and specialised methods exist for a wide range of flow types (e.g. shocks). However, it is not necessary to use such methods for geometrical configurations and pressure waves of the steepness considered herein. This is because it is possible to achieve high accuracy using standard numerical schemes provided that appropriate attention is paid to the design of the numerical grids and to carrying out suitable numerical checking processes.

For this study, the validation process had two distinct components, namely one to confirm the adequacy of the spatial grid size and the other to confirm the suitability of the temporal grid size. For simulations presented herein, a spatial grid size of $R_{\text {tun }} / 50=0 \cdot 1 \mathrm{~m}$ has been used over the whole of the solution domain inside the tunnel and close to the tunnel. An increased grid size has been used in the far field where the pressure fluctuations are much smaller than in the near field. The $0.1 \mathrm{~m}$ grid does not enable an exact representation of detailed flow structures close to sharp corners (because velocities tend to infinity at corners in an inviscid flow), but it is more than adequate for simulating wave propagation. This is true even at individual openings because the time scales associated with wave propagation through them are small in comparison with intervals of practical relevance to the resulting MPWs. The selected grid sizes have been validated by performing a grid independence study with progressive refinement of the grid size by factors of two. This study was undertaken with the full geometry shown in Figure 13 and the assessment was based on predicted values of $(a)$ mass-flow rates through the slots and $(b)$ pressures at key locations. Figure 14 shows a typical outcome using grid sizes of $0 \cdot 1$ and $0 \cdot 2 \mathrm{~m}$. It shows mass-flow rates through slots $1,4,7$ and 10 and the pressure history at the point where the reference line meets the tunnel axis (i.e. $r=0$ ). The differences are so small that no practical disadvantage would have been incurred if a larger grid size had been adopted. Nevertheless, to avoid any concern in this respect, only the smaller of the two grid sizes has been used in the simulations presented in the main body of the paper.

The other component of the numerical validation process was undertaken with a simpler geometrical configuration, namely
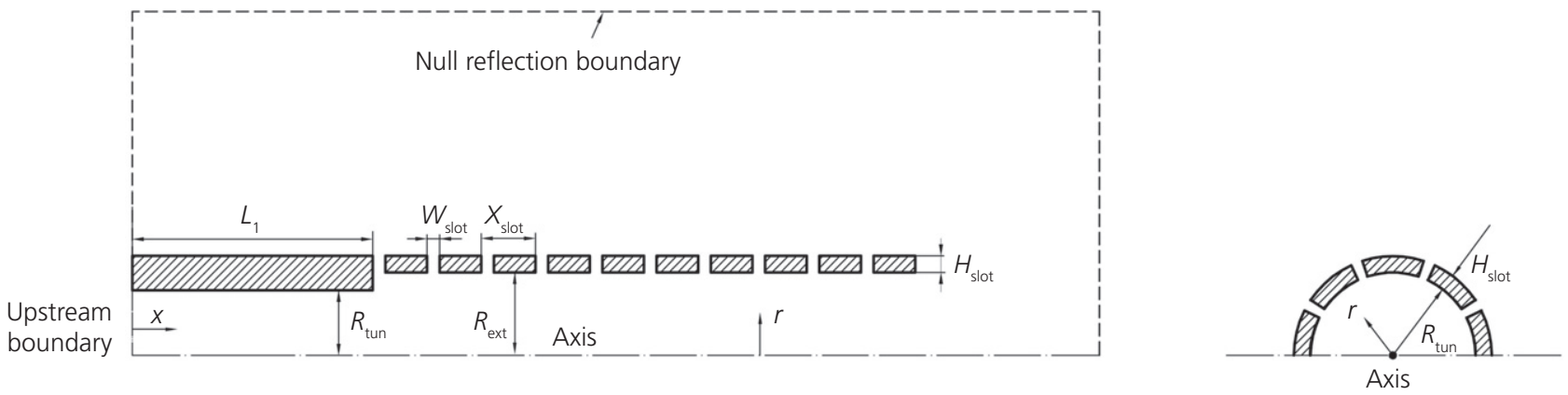

Figure 13. Tunnel geometry and solution domain 

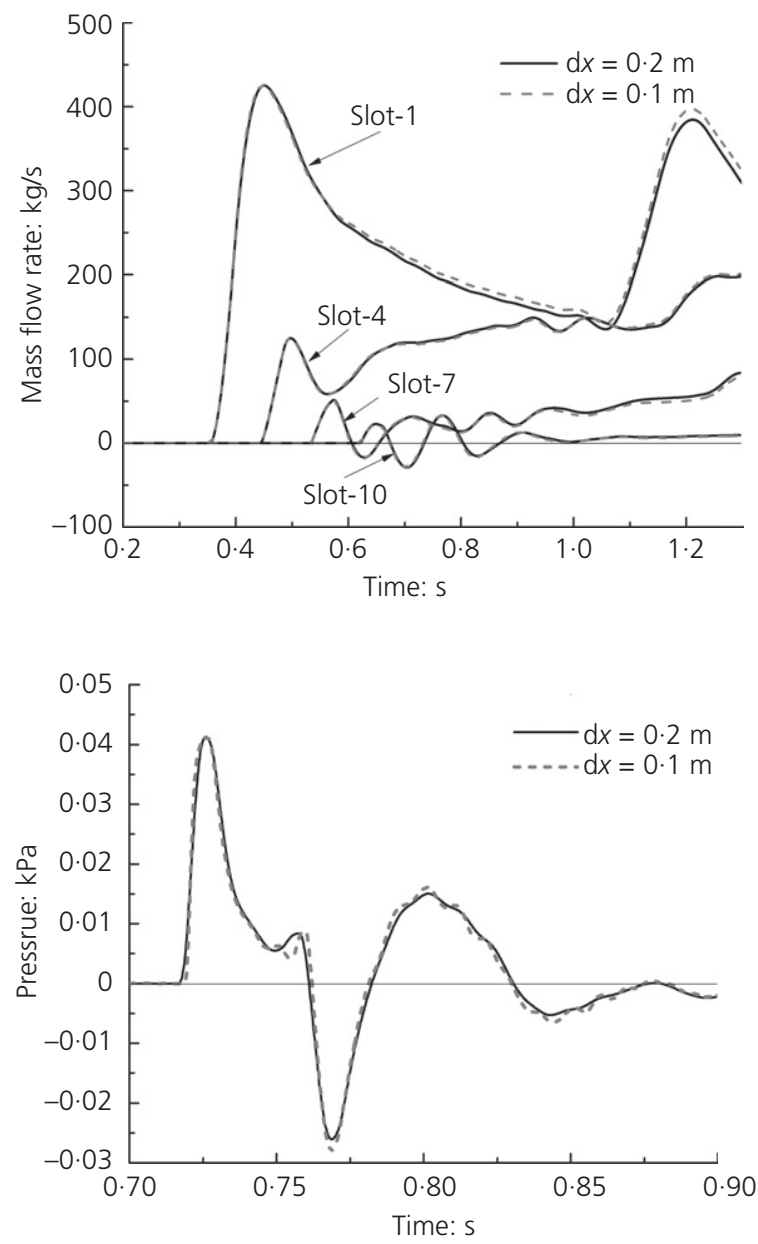

Figure 14. Influence of the numerical grid size: (a) mass-flow rates through slots; and $(b)$ pressure history at $(x, y)=(245,0)$

only the main tunnel upstream of the exit region in Figure 13. The purpose was to determine the most suitable time step for given spatial grid sizes. As the numerical scheme is implicit, stability can be achieved even for Courant numbers exceeding one, but it does not follow that adequate accuracy would also be achieved. Indeed, for the chosen numerical methodology, the optimised time step deduced from the validation study and used herein corresponds to a Courant number of $0 \cdot 3$. In this component of the validation study, a series of simulations has been undertaken with progressively increased steepness of the pressure rise at the upstream tunnel boundary until the additional (inertial) steepening of the resulting wavefront led to the development of near shock conditions close to the exit portal. By comparing the predictions with analytically exact steepening, it has been confirmed that the steepening process could be simulated with high accuracy over the main part of the wavefront with a broad range of Courant numbers.
The particular choice of 0.3 is made to minimise local numerical dispersion very close to the toe and heel of the induced wavefront. At these locations, the prescribed boundary condition enforces discontinuous rates of change of pressure and velocity that could be simulated with high accuracy only with specialised solution methods. However, the zones of significant numerical dispersion are highly local and of little, if any, practical significance. Wang et al. (2015a) presented graphical evidence of the suitability of corresponding simulations with 2D planar geometry. Herein, however, this is unnecessary because the suitability of the chosen grid is amply demonstrated by the comparisons presented in Figure 14.

\section{A1.1 Inviscid flow idealisation}

The methodology presented above ensures accurate numerical modelling of the analytical equations. For the solutions to be physically meaningful, it is additionally necessary for the analytical model to be a suitable approximation to the true flows. The most important simplification in this respect is the treatment of the flow as inviscid. The justification for this is conveniently considered in three stages, namely $(a)$ internal wave propagation, $(b)$ wave propagation through the exit portal and $(c)$ wave propagation through the lateral slots.

First, consider internal wave propagation. For this, inviscid approximations are highly realistic over short lengths of tunnel (small $L / D$ ) such as those considered herein. For instance, the frictional resistance to a steady flow with a mean velocity of $5 \mathrm{~m} / \mathrm{s}$ would imply a pressure difference of the order of $5 \mathrm{~Pa}$ in a $100 \mathrm{~m}$ long tunnel having a radius of $5 \mathrm{~m}$. This is very small in comparison with the wave amplitudes considered herein. Unsteady frictional resistance could be several times greater than quasi-steady resistance, but it is nevertheless far smaller than inertial pressure changes associated with the waves.

Next, consider wave propagation through the exit portal. When sufficient time is available for a well-established flow to develop from a duct, the outflow is in the form of a jet emerging into a zone of approximately uniform static pressure. The stagnation pressure at any point within the jet exceeds the laterally adjacent static pressure by almost $(1 / 2) \rho \boldsymbol{u}^{2}$, where $\rho$ denotes the air mass density and $\boldsymbol{u}$ is the local velocity. However, the time required for this well-established condition to develop greatly exceeds the wave times studied herein. The development process involves the creation of a vortex street originating at the edges of the portal. This phenomenon propagates more slowly than the jet velocity, which itself is typically two orders of magnitude smaller than the speed of wave propagation. Once again, therefore, viscous effects are of low importance during the early-stage flows of interest herein. 
Finally, consider wave propagation through the lateral slots and, to provide good focus, give special attention to the first slot encountered by the wavefront. This is the one through which the greatest velocities occur and it is also the one at which significantly elevated internal pressures exist for the longest time. As a preliminary step, consider quasi-steady flow through such a slot. If the upstream edges of the slot are sharp - as in figures herein - flow separation will occur at these edges and it will not reattach in the short distance available. Hence, the cross-sectional area of the vena contracta downstream of the slot will be considerably smaller than the geometrical area of the slot (perhaps around $40 \%$ smaller). Alternatively, if the upstream edges of the slot are well streamlined, separation will not have a strong influence and the vena contracta will be only slightly smaller than the geometrical area. For quasi-steady flows, the slot areas simulated herein should be interpreted as representative of vena contractas, not of true geometrical areas.

Now consider the opposite extreme of a rapidly varying flow such as that induced by a wavefront passing along the tunnel bore. The most important phase of this flow is when the rate of change is greatest (because it is rates of change of mass flow that induce external MPWs). In practice, this phase is too short for vorticity and separations to exert strong influence and, instead, the pressure will induce flow over the whole cross-sectional area of the slot, with little dependence on the sharpness of the corners. As a consequence, for the purposes of simulating the influence of the slots on MPW generation, the effective area of a slot is close to its full geometric area regardless of whether the upstream corners are streamlined.

It can be inferred from Figures 8(a), 8(c) 9(a), 9(c) that the greatest influence of the first slot occurs during a period of $\sim 0 \cdot 1 \mathrm{~s}$ after the toe of the incident wavefront reaches the slot. This is the period when the most rapid rates of change occur and the area of the vena contracta will be only slightly smaller than the geometrical outlet area, irrespective of streamlining. Thereafter, the flow will evolve towards quasi-steady behaviour and the simulated slot will be physically reasonable only if it is streamlined. For sharp-edged slots, the simulated rate of flow through the slot will be overestimated and, as a consequence, both it and the pressure along the region beyond the slot will be underestimated. However, the consequences of this are less strong than they appear at first sight because the reduced pressure will cause the corresponding outflow through the next slot to be underestimated. That is, the overall behaviour of a series of slots tends to be partially self-compensating. In summary therefore $(a)$ the predictions presented above should strictly be interpreted as applicable to streamlined slots with the same outlet areas as the simulated slots, but $(b)$ even for sharp-edged slots, the expected errors will not be sufficient to detract significantly from the practical value of the overall predictions.

For completeness, attention is now drawn to a loosely related case that has received much attention, namely compression wavefronts generated by trains entering tunnels with perforated entrance regions. In these cases, it is known that flow separation effects - and also inertial effects - can have a clear influence on the outcome for short regions (e.g. Howe, 2005). It should be noted, however, that there are big differences between this case and the exit region case. For instance, the overall time scale is determined by the time needed for the train nose to traverse the perforated region and, for today's high-speed trains, this is typically about three times as much as the time taken by a wave travelling the same distance. Thus, the relative importance of quasi-steady flows through slots (windows) is greatly increased. Moreover, entrance regions are designed to optimise the rate of increase of flow at the end of the region, not to minimise MPWs emitted from the slots. This behavioural difference is so strong that impressive agreement with physical measurements for long regions can be obtained with 1D models that wholly neglect inertial influences of flows through the slots (e.g. Vardy et al., 2015).

\section{REFERENCES}

ANSYS (2011) ANSYS Fluent UDF Manual, Release 14.0. ANSYS, Inc, Canonsburg, PA, USA.

Aoki T, Vardy AE and Brown JMB (1999) Passive alleviation of micro-pressure waves from tunnel portals. Journal of Sound and Vibration 220(5): 921-940.

Brown JMB and Vardy AE (1994) Reflections of pressure waves at tunnel portals. Journal of Sound and Vibration 173(1): 95-111.

Fukuda T, lida M and Murata K (2001) Distortion of compression wave propagating through slab track tunnel with side branches. Quarterly Report of RTRI 42(2): 71-76.

Fukuda T, Ozawa S and lida M (2006) Distortion of compression wave propagating through very long tunnel with slab tracks. JSME International Journal, Series B 49(4): 51-57.

Fukuda T, Saito S, lida M, Kurita T and Ozawa S (2013) Countermeasure against the micro-pressure wave by a shelter linking neighbouring tunnels. Proceedings of the 15th International Symposium on Aerodynamics, Ventilation and Fire in Tunnels, Barcelona, Spain, pp. 539-552.

Hieke M, Gerbig CH, Tielkes T and Degen KG (2011) Assessment of micro-pressure wave emissions from high-speed railway tunnels. Proceedings of the 9th World Congress on Railway Research: Challenge B: An Environmentally Friendly Railway, Lille, France, CD-ROM.

Howe MS (2005) On the role of separation in compression wave generation by a train entering a tunnel hood with a 
window. IMA Journal of Applied Mathematics 70(3): 400-418.

Howe MS, lida M and Fukuda T (2003) Influence of an unvented tunnel entrance hood on the compression wave generated by a high-speed train. Journal of Fluids and Structures 17(6): 833-853.

Kim H, Kweon YH and Aoki T (2004) A new technique for the control of a weak shock discharged from a tube. Journal of Mechanical Engineering Science, Part C 218(4): 377-387.

Matsubayashi K, Kosaka T and Kitamura T (2004) Reduction of micro-pressure wave by active control of propagating compression wave in high speed tunnel. Journal of Low Frequency Noise, Vibration and Active Control 23(4): 259-270.

Ozawa S and Maeda T (1988) Tunnel entrance hoods for reduction of micro-pressure wave. Quarterly Report of RTRI 29(3): 134-139.

Ozawa S, Maeda T, Matsumura T et al. (1991) Counter-measures to reduce micro-pressure waves radiating from exits of Shinkansen tunnels. Proceedings of the 7th International Symposium on Aerodynamics and Ventilation of Vehicle Tunnels, Brighton, UK. BHR Group, Bedford, UK, South Coast of England, pp. 253-266.

Raghunathan SR, Kim HD and Setoguchi T (2002) Aerodynamics of high-speed railway train. Progress in Aerospace Sciences 38(6-7): 469-514.
Rudinger G (1955) On the reflection of shock waves from an open end of a duct. Journal of Applied Physics 26(8): 981-993.

Rudinger G (1957) The reflection of pressure waves of finite amplitude from an open end of a duct. Journal of Fluid Mechanics 3(1): 48-66.

Sockel $\mathrm{H}$ and Pesave P (2006) The reduction of micro pressure waves by baffles. Proceedings of the 9th International Symposium on Aerodynamics and Ventilation of Vehicle Tunnels, Portoroz, Slovenia. BHR Group, pp. 804-817.

Sugimoto N (1993) Shock-free tunnel for future high-speed trains. Proceedings of International Conference on Speedup Technology for Railway and Maglev Vehicles, Yokohama, Japan, Paper PS3-3, Vol. II, pp. 284-292.

Vardy AE, Sturt R, Baker CJB and Soper D (2015) The behaviour of long entrance hoods for high speed rail tunnels. Proceedings of the 16th International Symposium on Aerodynamics, Ventilation and Fire in Tunnels, Seattle, USA. BHR Group, Bedford, UK, pp. 449-466.

Wang $H$, Vardy AE and Pokrajac D (2015a) Pressure radiation from a perforated duct exit region. Journal of Sound and Vibration 351: 29-42.

Wang H, Vardy AE and Pokrajac D (2015b) Perforated exit regions for the reduction of micro-pressure waves from tunnels. Journal of Wind Engineering and Industrial Aerodynamics 146: 139-149.

\section{WHAT DO YOU THINK?}

To discuss this paper, please email up to 500 words to the editor at journals@ice.org.uk. Your contribution will be forwarded to the author(s) for a reply and, if considered appropriate by the editorial panel, will be published as discussion in a future issue of the journal.

Proceedings journals rely entirely on contributions sent in by civil engineering professionals, academics and students. Papers should be 2000-5000 words long (briefing papers should be 1000-2000 words long), with adequate illustrations and references. You can submit your paper online via www.icevirtuallibrary.com/content/journals, where you will also find detailed author guidelines. 